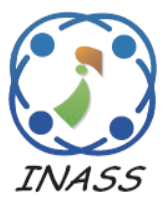

\title{
Channel and Relay Assignment Using Kruskal Algorithm in LTE Networks
}

\author{
Santhakumar Rathnasamy $^{1} \quad$ Amutha Prabha Nagarajan ${ }^{1 *}$ \\ ${ }^{1}$ School of Electrical Engineering, Vellore Institute of Technology University, India \\ * Corresponding author's Email: amuthaprabha@vit.ac.in
}

\begin{abstract}
The paper deals with the study of throughput maximization by relay selection and assignment along with channel and subcarrier allocation. In particular, Kruskal algorithm is applied to solve radio resource allocation problem in wireless communication. Solution for joint optimization of carrier and relay is proposed in this work. Graphical theory approach is examined for solving the problem optimally in a given polynomial time. Total network throughput performances variations are analysed through numerical simulation for various combinations of transmit power nodes and relay nodes. It is also demonstrated in this paper the improvement in sum rate against Greedy's algorithm and Prim's algorithm using proposed Kruskal algorithm. Simulation results obtained show that the Kruskal algorithm based approach around 10\% to 22\% improvement in Sumrate over the fixed subcarrier pairing for a given source power for a carrier and number of relays.
\end{abstract}

Keywords: Orthogonal frequency division multiplexing, Relay, Sub-carrier, Kruskal algorithm, Graph theory, Throughput.

\section{Introduction}

Consumer demand for radio resources has been growing enormously, fuelled by smart devices such as laptops, mobile phones and tablets. This poses series strain on conventional wireless network systems. To meet the growing demands and reduce the complexity of deployment of cellular networks, many techniques have been introduced. Cooperative relay network is one the key technologies that is getting spotlight to satisfy the booming volume of radio resources. The reason is that, it could exploit different diversity schemes and user cooperation to extend the coverage and improve the performance of the system.

Recently, strategy for cooperative transmissions gets prominent attention in emerging wireless networks. The relay transmission schemes inherently adapt wireless transmission broadcasting nature, spatial diversity and multiuser diversity for the cooperative transmissions algorithm implementation. Most of the published transmission schemes employing cooperative transmission concentrate on link quality improvement, whereas works in radio resource allocation have not yet considered suitable algorithms for better resource management. Increasing present day demand for variety of services and limited availability of radio resources, it is essential to develop suitable schemes and algorithms for cooperative relay transmission mechanisms.

In the early stages of relay networks, coding schemes that exploit node cooperation are used for networks using relay for transmission. Basically the relays decode and forward or compress and forward their output to other nodes. It has been found that, if relay is nearer to the source node and phase information is available, the relay assisted schemes achieves the ergodic capacity [1]. Decode and Forward (DaF) and amplify and forward $(\mathrm{AaF})$ strategies for opportunistic relaying is considered for average power constraint. It is found that opportunistic $\mathrm{AaF}$ with average power constraint is better compared to AaF with equal power constraint and $\mathrm{DaF}$ is outage optimal [2] in cooperative relay networks. To maximize the throughput in cooperative relay networks, stochastically varying network traffic flow is considered. Moreover, jointly 
optimizing routing, scheduling and resource allocation for optimal control of network throughput is also discussed in [3]. Also joint optimization of power, channel pairing, and assignment of user over channels has been discussed in the past to maximize the weighted sum-rate in single relay networks [4].

The impact of power allocation in OFDM based single relay networks is investigated for mutual information between source and node [5]. Resource allocation for OFDM based relay networks with user pairs is demonstrated to maximize the sum rate among the user pairs. Also to maximize the throughput, optimal coordination between relay and sub-carrier assignment is required [6]. The idea of relay selection by allowing different relays to cooperate has been proposed earlier. SNR optimal relation scheme with much reduced complexity to achieve full diversity is studied in [7]. Relay selection based on instantaneous base stations to user channels, target throughput of the users and channels between users is also discussed in [8]. Using the instantaneous channel conditions of source to relay and relay to source nodes, one of the best relay selection schemes has been proposed in [9]. Performance of relay nodes with game theoretic framework is investigated for spectral efficiency maximization [10].

To alleviate the spectral efficiency loss, a transmission scheme has been proposed in decode and forward relaying networks [11]. In [12], authors proposed a joint power allocation and strategy selection scheme for half duplex decode and forward relay system. Power allocation for secure communication [13] in cooperative networks and also joint power allocation for multi-relay OFDM system with less computational complexity are proposed [14]. Moreover, joint optimization of power, channel user assignment and channel pairing is proposed to maximize the throughput in cooperative single relay systems. While optimizing the throughput in these works, common relaying methods are considered in addition to power constraints on individual as well as aggregate transmitters [15].

To estimate the error propagation in relaying, threshold based relaying scheme is examined. The relays forward the bits if it is correctly decoded by the relays. This accounts for studying the bit error rate performance in relaying schemes [16]. Other fundamental issue in multihop relaying is the packet delay. An effective optical and heuristic channel assignment scheme to overcome the problem of packet delay in multihop cellular networks has been proposed [17]. Recently total throughput is maximized using Prim's algorithm by considering subcarrier allocation and assignment along with channel and relay assignment. While invoking the optimization using prims algorithm, graphical theory approach is used to optimize the throughput in polynomial time [18].

In most of the previous work on relay selection, relay assignment and channel assignment concentrates on power allocation, improving link quality with channel state information, maximization of throughput without jointly assigning channel and relay. It has been investigated from the recent research works, that radio resource allocation with joint optimization of number of relays and sub-carrier pairing is a vital problem for guaranteed sum rate in relay networks. In this research article, our idea is to propose a solution for jointly assigning relay, carrier and channel for higher Sumrate using Kurskal algorithm.

The disadvantage of the proposed approach compared to the prim's algorithm [18] is that it takes more time for optimal solution. Moreover, Prim's generate connected Minimum Spanning Tree (MST) but in the case of Kruskal the MSTs may not be connected. If it is a disconnected node, Kruskal forms a collection of MSTs which further helps in sorting the nodes. Another important fact of this Kruskal's algorithm is that it can be easily implemented for sparse networks.

The obtained results indicate that the complexity of proposed approach is much less than the other counter parts like fixed subcarrier paring and Greedy algorithm. In case of fixing the relays and studying the Sumrate, the work implemented in this paper produces a healthy improvement which can support the present day throughput demands.

The rest of the paper is organized as follows. Section 2 describes the system model and in section 3 graph based optimization for relay system is explained. Performance evaluations are given in section 4 and finally section 5 concludes the paper.

\section{Resource allocation system model}

The system model consists with an OFDMbased wireless network shown in Figure 1. Exchange of information between $\mathrm{K}$ numbers of user pairs through $\mathrm{R}$ number of relays are considered in the system. Any user can exchange the information with its pair through any one of $\mathrm{R}$ relays. The shadowing effect is nullified by not permitting direct communication between any two sources. The source and relay is communicating in half duplex mode. A non-transparent centralized scheme for relay allocation is followed to control the exchange 


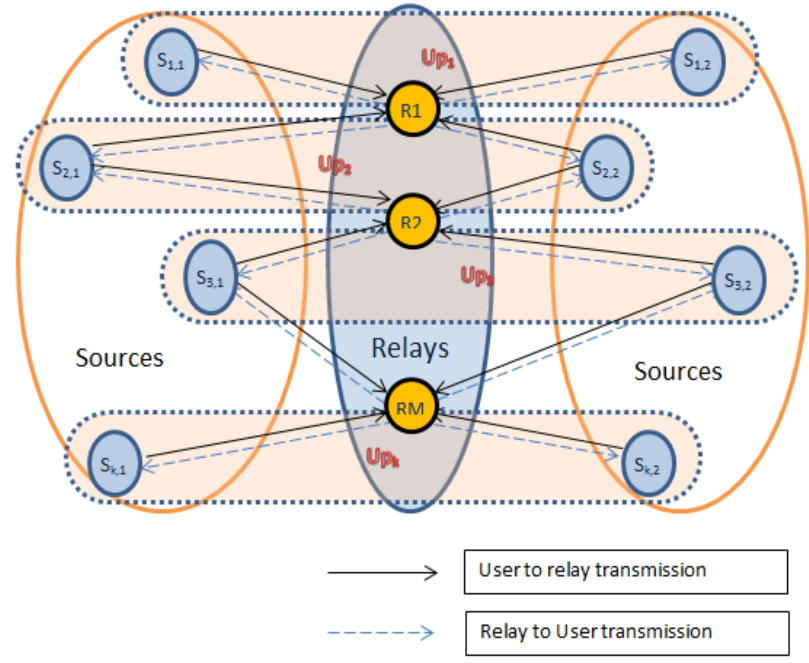

Figure.1 OFDM wireless network model with relay and user pair

of information between sources and relay [19]. The relays operate in two-way relaying mode and moreover, it forward the information supplied by the source while performing amplification with constant gain. The signals are modelled in this work using shadowing and large scale path loss model. The fading of the signals are assumed to follow frequency selective fading. We consider that the channel estimation is perfectly available to the relays and sources in the network. Also, the network communicates in slow fading environment but at the same time the links are communicating through independent fading channels. The white noises are considered as independent circular symmetric complex Gaussian random variables and the transmit power of all subcarriers are uniformly distributed.

Two phases of communication between pair of users are considered to model the system. That is, a array for subcarriers $\mathrm{N}=\{1,2, \ldots\}$, an array for relays $R=\{1,2, \ldots\}$, and another array for user pairs $S=\{1,2, \ldots\}$ are considered in the proposed model. Let $S_{\mathrm{k}, 1}$ and $\mathrm{S}_{\mathrm{k}, 2}$ are the pair of sources associated with $\mathrm{k}^{\text {th }}$ set user pair. The user pair $\mathrm{k}$ is exchanging information between them through $\mathrm{r}^{\text {th }}$ relay. The two phases are described as follows

\section{Phase 1 - Multiple Accesses (MAC):}

The source $\mathrm{Sk}, 1$ user transmitter sends the signal to relay $r$. The communication is established using subcarriers which are non-overlapping to avert interference. The alphabet $\mathrm{m}$ denotes the subcarrier used in phase one. The interference emanating from intra-pairs are considered as self-interference. This self-interference is modelled as back-propagated interference and is easily cancelled after two-way relaying.

\section{Phase 2 - Broadcast (BC):}

Here the received signals are amplified and broadcast to $2 \mathrm{~K}$ user by relay $\mathrm{r}$. By using non overlapping subcarriers $\mathrm{n}$ for each relay, inter relay interference will be avoided. Two or more pair of subcarriers is used by each relay.

To transmit the signal to relay $r$ from the user $\mathrm{S}_{\mathrm{k}, 1}$ a subcarrier $\mathrm{m}$ is assigned. To transmit from relay $r$ to the user $S_{k, 2}$ a subcarrier $n$ is used. The sum rate for user pair $\mathrm{k}$ over subcarrier pair $(\mathrm{m}, \mathrm{n})$ through relay $\mathrm{r}$ can be achieved using the following relation,

$$
\begin{gathered}
R_{k, r, m, n}=\frac{1}{2} \log _{2}\left(1+\frac{\gamma_{k_{1}, r, m} \gamma_{r, k_{2}, n}}{1+\gamma_{r, k_{2}, n}+\gamma_{k_{1}, r, m}+\gamma_{k_{2}, r, m}}\right)+ \\
\frac{1}{2} \log _{2}\left(1+\frac{\gamma_{k_{2}, r, m} \gamma_{r, k_{1}, n}}{1+\gamma_{r, k_{1}, n}+\gamma_{k_{1}, r, m}+\gamma_{k_{2}, r, m}}\right)
\end{gathered}
$$

In the above equation, the parameter $\gamma \mathrm{i}, \mathrm{j}, \mathrm{n}$ represents the instantaneous SNR measured at jth node in the nth subcarrier transmission. The noise variance in each node is assumed to be unity. To represent mathematically the subcarrier allotment, binary variables $B_{k, r, m, n} \in\{0,1\}$ are introduced. In the binary variables, $\mathrm{k}$ represents user pair, $r$ denotes relay, and the subcarrier index is represented as $m$ and $n$. The pairing of the subcarriers are denoted by $B_{k, r, m, n}=1$. This is equivalent to a subcarrier $m$ in the initial phase is paired with another carrier $n$ in the next phase. The pairing is assisted by relay $r$ for user a pair $k$. For other pairing conditions, the binary variable $B_{k, r, m, n}$ is equal to zero. Every subcarrier in each phase is assigned to one user pair and one relay to nullify interference. The constraints should be satisfied by the binary variable $\left\{B_{k, r, m, n}\right\}$ is given as

$$
\begin{aligned}
& \sum_{k \in K} \sum_{r \in R} \sum_{n \in N} B_{k, r, m, n} \leq 1, \forall_{m} \in N \\
& \sum_{k \in K} \sum_{r \in R} \sum_{m \in N} B_{k, r, m, n} \leq 1, \forall_{n} \in N
\end{aligned}
$$

From the above equations, the system sumrate is maximized by pairing the subcarriers optimally in the two phases. To maximize the throughput further, best user pairs and the best relays are also required. From Eqs. (1), (2) and (3), the mathematical formula for assignment of relay and subcarrier pairing is arrived as

$$
\max \sum_{k \in K} \sum_{r \in R} \sum_{m \in N} \sum_{n \in N} R_{k, r, m, n} B_{k, r, m, n}
$$




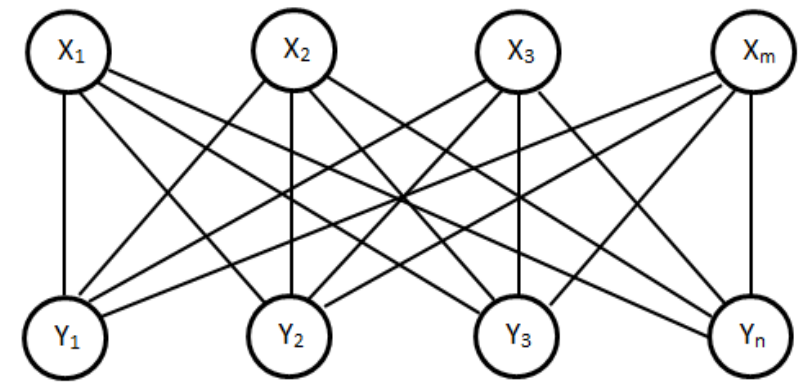

Figure. 2 Bipartite graph approach

\section{A graph based optimization}

An optimization algorithm for relay selection and subcarrier pairing to deduce the optimal solution is given in Eq. (4). The Eq. (4) requires long search operation to find the optimal solution. When $\mathrm{K}, \mathrm{R}$ and $\mathrm{N}$ increase, optimal solution will not obtained due to the complexity of the problem and also increases in exponential of the solution. Algorithm based on graphical approach to find the weighted sum of all rates is developed to alter the objective function of Eq.(4). The modified objective function of Eq.(4) is not changing the optimum solution. The proposed graph based algorithm determines the subcarrier pair $(m, n)$ which is the maximum weighted pair as follows.

$$
R(m, n)=\max _{k \in K . r \in R} R_{k, r, m, n}
$$

The variables $\mathrm{u}$ and $\mathrm{v}$ are introduced to denote the maximum number of user pair and relay node linked for each subcarrier pair $(m, n)$ in Eq. (5). Consequently, without loss of optimality, we can convert the existing approach in the Eq.(4) to the following new problem which is now given as Eq. (6).

$$
\begin{array}{cl}
\max \sum_{m \in N} \sum_{n \in N} R(m, n) B_{u, v, m, n} \\
\text { s.t. } & \sum_{n \in N} B_{u, v, m, n} \leq 1, \forall_{m} \in N, \\
& \sum_{m \in N} B_{u, v, m, n} \leq 1, \forall_{n} \in N,
\end{array}
$$

As stated above, the problem in Eq. (6) is a simplified problem. The maximum weighted bipartite matching (MWBM) problem can very well now approximated to the derived problem in Eq. (6). Thus the division of nodes in a bipartite is divided into two disjoint set and every set of edges connected to another set. The cardinality of the both the sets are same as per the balanced bipartite graph. A matching is a set of mutually disjoint edges, when say any two edges do not share a common node. Fig. 2 shows that in the bipartite graph, every node is matched and the matching is also perfect. Perfect matching is the special case of matching. There are couple of vertices $\mathrm{V}_{1}=\left[\mathrm{X}_{1}, \mathrm{X}_{2}, \ldots \mathrm{Xm}\right]$ and $\mathrm{V}_{2}=\left[\mathrm{Y}_{1}\right.$, $\left.\mathrm{Y}_{2}, \ldots . \mathrm{Yn}\right]$ in the balanced bipartite graph $\mathrm{G}=$ $\left(\mathrm{V}_{1} \times \mathrm{V}_{2}, \mathrm{E}, \mathrm{W}\right) . \mathrm{N}$ is the set of subcarriers in the MAC phase and the $\mathrm{BC}$ phase respectively.

Fig. 2 also indicates the set of edges $E$ that join all possible vertices pairs in the set of vertices. In each phase, the subcarrier $\mathrm{N}$ is shared giving raise to $\left|\mathrm{V}_{1}\right|=\left|\mathrm{V}_{2}\right|=|\mathrm{N}|=\mathrm{N}$. The set of edges $|\mathrm{E}|$ reduces to $\mathrm{N}_{2}$. The cardinality of a set is represented as |.| and the weighting function is $\mathrm{W}$. The weighing function belonging to each set of edges is real quantity. To get optimum achievable rate over the joined vertices, each edge is allocated with a weight. Thus the weight is represented as

$$
W_{(m, n)}=R(m, n)
$$

where $R(m, n)$ is defined in Eq.(5).

The weighting process is calculated across all edges of the graph. So, the total complexity of above Eq. (7) is polynomial. Thus the graph represents to find the following equivalences: First a pair of subcarrier matched in the MAC and BC phases, secondly a matching implies the subcarrier assignment in each phase defined in Eq. (2) and Eq. (3). Also, the optimal user pair and relay determination for subcarrier pair is equivalent to assigning weight for each edge in E. Meanwhile, the consecutive of the kruskal algorithm of subcarrierpairing based subcarrier assignment and relay selection in multi-relay multi-pair two-way relaying networks for the total throughput maximization is equivalent to finding a perfect matching $F$ is subset of $\mathrm{E}$ in $\mathrm{G}$ so that the sum weights of $\mathrm{F}$ is maximum. This is called as MWBM problem which is NPComplete and is given in the Eq.(8). The Eq.(8) is written as

$$
\max _{F \subseteq E} \sum_{(m, n) \in F} W_{(m, n)}
$$

The above Eq. (8) is equivalent to Eq. (5). Thus the original problem in the Eq. (4) is simplified in the Eq.(5). The problem is again converted as a problem of MWBM in the Eq. (6) without loss of optimality.

\subsection{Kruskal algorithm}

It is one of the renowned greedy algorithms which are a key to provide an optimal solution to many problems. This algorithm gives benefits in estimating the least weighted edge that joins two nodes in directed graphs. This results in construction of minimum spanning tree with an addition of increased cost arcs at each and every step. 


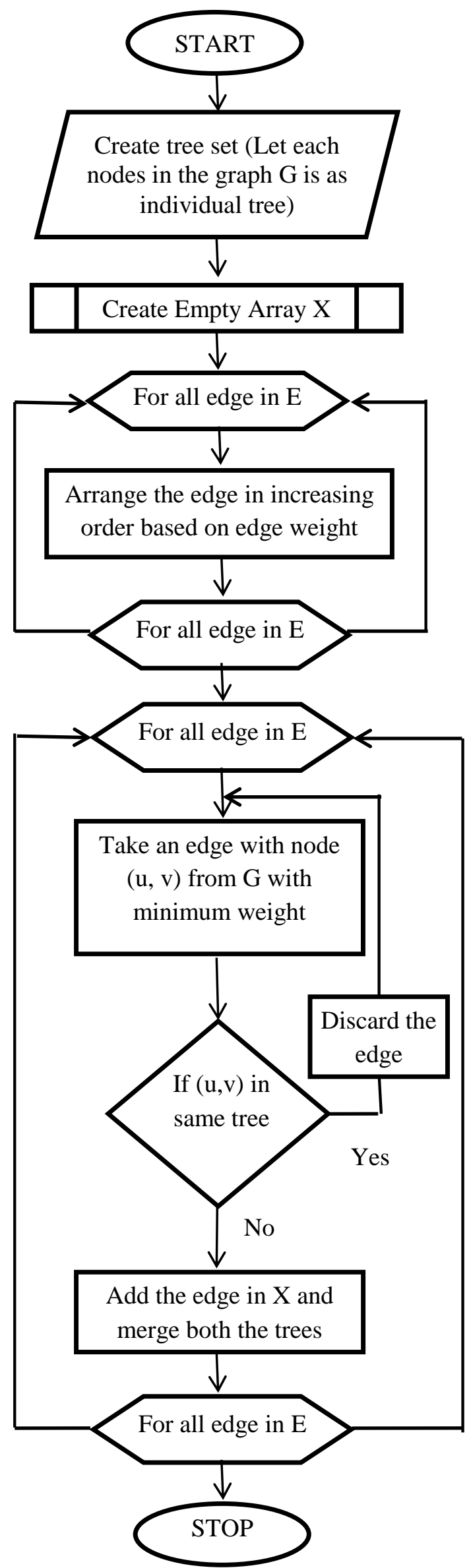

Figure.3 Flow charge for kurskal's algorithm
"Minimum spanning tree" is a graph theory where a graph or tree is erected by connecting all the nodes without forming cycles or individual loops among three or more nodes with minimum cost. Initially, an unsolved tree is set as an input to this algorithm with all nodes connected and some weights on it. On implementing the algorithm the edge with the least weightage is drawn as the output in output graph followed by second least weighted edge and so on. Each and every node is drawn at the output graph such that all the nodes are present in the original graph without formation of cycles. The spanning tree for that graph would be a subset of those paths that has no cycles but still connects to every node. Some of the applications of spanning tree are Cartography, Clustering and Mechanical / Electrical / Computer Networks.

Despite having enormous applications the algorithm are mostly used to minimize the total cost of wiring in electrical network. There might be a problem in choosing the best algorithms in minimum spanning tree algorithms like Prims, Bipartite graph etc. Kruskal algorithm has got a special feature that it works much better in certain typical situations (sparse graphs) as simplest data structures are used in this algorithm. It provides best performance when edges are already sorted and if they can be sorted in linear time.

The input graph $\mathrm{G}=(\mathrm{V}, \mathrm{E})$ is taken, where $|\mathrm{V}|=$ $\mathrm{n}$ is the set of node, $|\mathrm{E}|=\mathrm{m}$ is the set of edge and a set of weights $w$ that corresponds to each edge e $\epsilon$ E. The MST X starts out as an empty tree, and each node is itself a tree. The for-loop in line 3 examines each edge $(\mathrm{u}, \mathrm{v})$ in order of weight, from lowest to highest.

At each time, we check whether endpoints $u$ and $\mathrm{v}$ belongs to the same tree. If they belong to the same tree, we discard such edge; otherwise we add it to $\mathrm{T}$ and merge $\mathrm{u}$ and $\mathrm{v}$. The algorithm is as follows.

\section{Kruskal's Algorithm}

1. for all $\mathrm{u} \in \mathrm{V}$ :

Create a tree set containing node $(\mathrm{u})$

2. Create empty tree $(X=\{\})$

3. Sort the edges $E$ for all edges $e(u, v) \in E$ based on its weight in the graph G. Bubble sorting algorithm is used to arrange the edges in increasing fashion.

4. Select any edge e $(u, v)$ from $E$

5. if node $\mathrm{u}$ and node $\mathrm{v}$ are in different tree(not in same tree): add edge $\mathrm{e}(\mathrm{u}, \mathrm{v}) \in \mathrm{X}$

6. merge the sets containing $\mathrm{u}$ and $\mathrm{v}$ (union $(\mathrm{u}, \mathrm{v})$ ) 
7. else discard the edge

8. repeat steps 4,5,6, and 7 until all edges are selected

\section{Simulation results}

The results obtained are based on fixed subcarrier pairing scheme. By using Kruskal algorithm optimum solution is obtained due to optimal subcarrier mapping. One subcarrier signals in the MAC phase is subsequently transmitted in the $\mathrm{BC}$ phase by a relay on the same subcarrier. The greedy algorithm can solve the problem optimally. The greedy algorithm is having less complexity for selection of relay and optimal user pair for each subcarrier. The simulation results are plotted for greedy algorithm [6], Prims algorithm [18] and kruskal algorithm. Uniform distribution is considered for selection of source and relay nodes during simulation. Random distribution is considered for source, destination and relay nodes which are shown in Fig.4. The parameters used for simulation is given in Table 1 .

In this work, the path loss model is taken from [6]. The path loss exponent of the proposed work is assigned as 4 . The log-normal shadowing standard deviation is assigned as $5.8 \mathrm{~dB}$. Small scale fading in this proposed model is modelled using multi-path Rayleigh fading process. While modelling the Rayleigh fading process exponentially decaying power delay profile is assumed and it is having a maximum delay spread of $5 \mu \mathrm{s}$. The Doppler spread is taken as $5 \mathrm{~Hz}$. To perform simulations, sums of 2000 channel realizations were generated and all the channel realizations are independent. Each channel realizations are associated with different node locations. The number of subcarriers are $\mathrm{N}=32$. All sources have the same maximum power constraints, while all relays satisfying $\mathrm{P}_{\mathrm{r}}=\mathrm{P}_{\mathrm{k} 1}+3 \mathrm{~dB}=\mathrm{P}_{\mathrm{k} 2}+3 \mathrm{~dB}$ (per-subcarrier) for all $\mathrm{r}$ and $\mathrm{k}$.

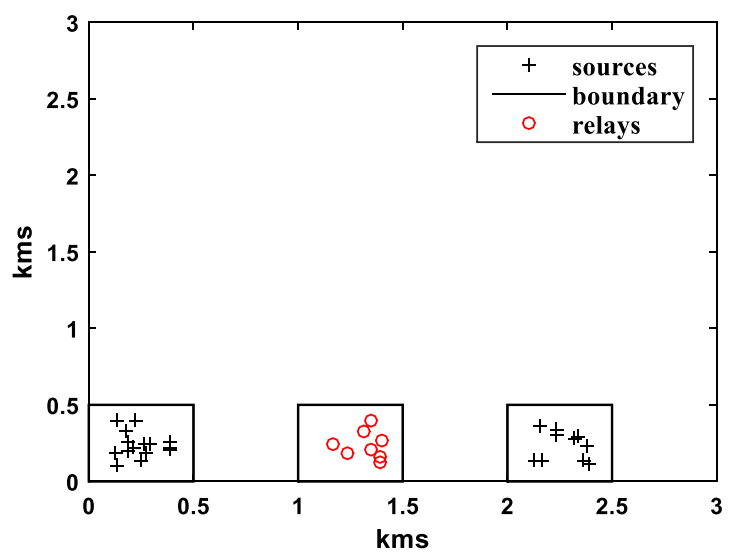

Figure.4 Two dimension plane of user and relay
Table 1. System simulation parameters

\begin{tabular}{|c|c|}
\hline Parameters & $\begin{array}{c}\text { Corresponding } \\
\text { Values }\end{array}$ \\
\hline Number of Source Nodes & 10 \\
\hline Number of Destination Nodes & 10 \\
\hline Number of Relay Nodes & 8 \\
\hline Number of Subcarriers & 64 \\
\hline Doppler Spread & $5 \mathrm{~Hz}$ \\
\hline Path Loss Exponent & 4 \\
\hline Maximum Delay Spread & $5 \mu \mathrm{s}$ \\
\hline No. of Channel Realizations & 2000 \\
\hline
\end{tabular}

To show the performance improvement, fixed subcarrier pairing scheme is considered as benchmark scheme. As in [6], user pair transmits signals in MAC phase through one carrier is forwarded in the BC phase on the same carrier by a relay i.e., $m=n$. Instead of looking for best subcarrier pairing, same subcarrier is used in MAC phase and $\mathrm{BC}$ phase. Now the problem reduces to selection of optimal user pair and relay for each subcarrier to achieve throughput maximization. The optimal user pair and relay selection is achieved by the greedy algorithm. That is each subcarrier $\mathrm{m}$ shall be assigned a user pair and the relay which is given as

$$
(\mathrm{u}, \mathrm{v})=\arg \max _{\mathrm{k} \in \mathrm{K}, \mathrm{r} \in \mathrm{M}} \mathrm{R}_{\mathrm{k}, \mathrm{r}, \mathrm{m}, \mathrm{n}} \text {. }
$$

Fig.5 demonstrates the sum-rate performance against the source power when five users and four relay network is considered. It can be noticed that there is $22 \%$ improvement in the throughput of the system by using the proposed kruskal algorithm when compared to fixed pairing scheme. Moreover, it is also easily inferred from the Fig. (5) that the proposed kruskal algorithm achieves a total throughput improvement of about $12 \%$ over the classical Greedy Algorithm. The observed performance improvement in the proposed algorithm over the existing algorithms is that the proposed algorithm sorting the edges in ascending order. This sorting helps the system to select the best matching pair thereby increasing Sumrate.

Among Greedy, Prims and Kruskal algorithms, Kruskal algorithm shows better results. Compared to Prims and previous max weighted bipartite graph algorithms, the Kruskal algorithm provides higher sum-rate which is shown in the Fig.6. Complexity of fixed pair subcarrier and Graph based greedy algorithm is $\mathrm{O}(\mathrm{KRN})$ and $\mathrm{O}(\mathrm{KRN} 2+\mathrm{N} 3)$ respectively. The complexity of the proposed algorithm is $\mathrm{O}(\mathrm{KRN} \log \mathrm{KRN})$, whereas Prims [18] has $\mathrm{O}(\mathrm{KRN} 2)$. In prims algorithm, it can be noticed 


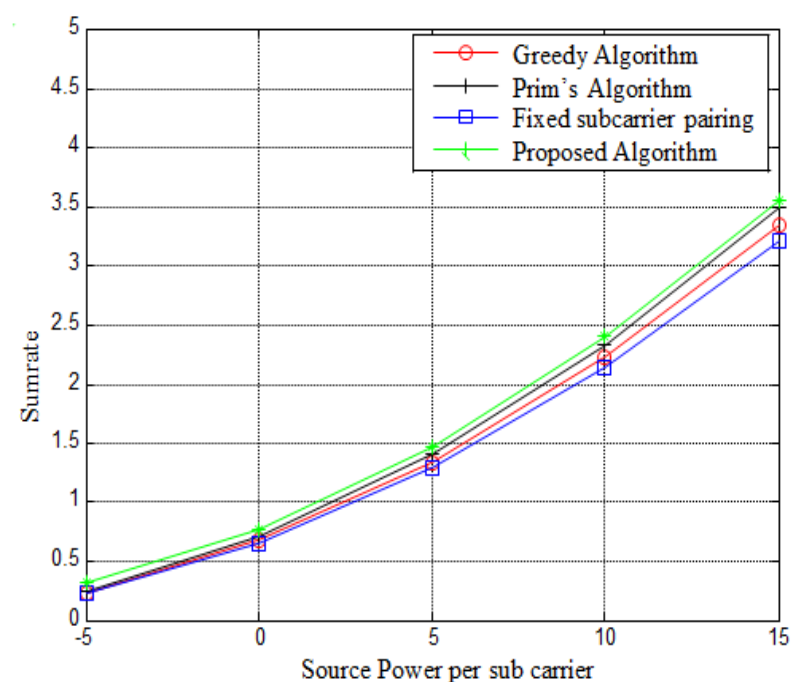

Figure. 5 Performance variations

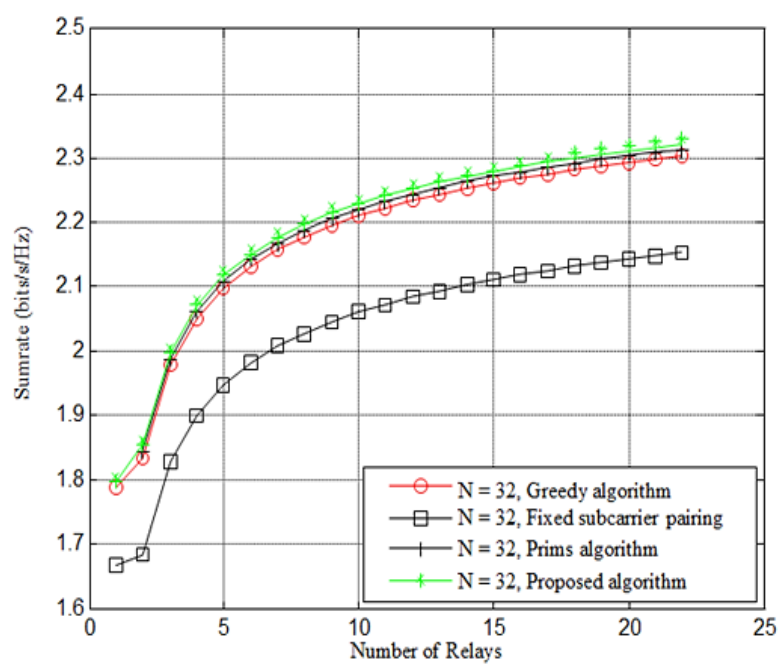

Figure. 6 Effects of the number of relays, where $N=$ $32, K=10$, and $P k 1=10 \mathrm{~dB}$

the results obtained was proved to be better compared to fixed subcarrier [6]. But, the proposed algorithm provides better results in typical situations as it uses sparse graph for computation and simpler data structures.

Fig.7 provides the throughput for various number of subcarrier pairing. The adaptive subcarrier pairing considered for simulation in the work are $\mathrm{N}=16, \mathrm{~N}=32$ and $\mathrm{N}=64$. Marked improved in the system throughput is observed when number of subcarrier pairing is $\mathrm{N}=16$.

In addition to fixing the subcarrier pairing, the increment in throughput is also high when the number of relays got increased. This phenomenon of Sumrate increment is common when the number of subcarriers are changed to $\mathrm{N}=32$ and $\mathrm{N}=64$.

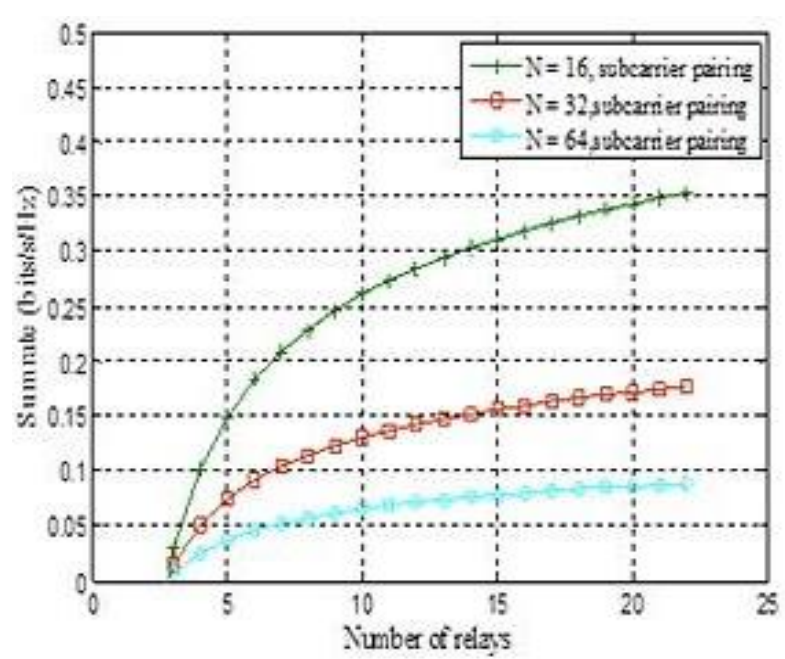

Figure. 7 Effects of the number of relays with different subcarrier

\section{Conclusion}

The work concludes that kruskal algorithm maximize the sum rate. In the proposed approach, subcarrier-pairing and relay selection using kruskal algorithm is studied to maximize the throughput in LTE networks. The proposed kruskal algorithm for optimization of subcarrier and channel using bipartite graph produces the solution in polynomial time. The results clearly showed that the Kruskal algorithm based joint optimization can be applied to the next generation networks which normally require higher data rates. The proposed approach required less power per node compared to other existing approaches considered in this article.

This work can easily be extended to networks having multiple antennas at both the relays and the nodes. This Kruskal algorithm can also be extended to jointly optimizing the power per node along with subcarriers.

\section{References}

[1] G. Kramer, M. Gastpar, and P. Gupta, "Cooperative Strategies and Capacity Theorems for Relay Networks", IEEE Transactions on Information Theory, Vol.51, No.9, pp.3037-3063, 2005.

[2] A. Bletsas, H. Shin, and M. Z. Win, "Cooperative Communications with Outage-Optimal Opportunistic Relaying", IEEE Transactions on Wireless Communications, Vol.6, No.9, pp.34503460, 2007.

[3] E. M. Yeh and R. A. Berry, "Throughput Optimal Control of Cooperative Relay Networks", IEEE Transactions on Information Theory, Vol.53, No.10, pp. 3827-3833, 2007.

[4] T. S. Chang, K. T. Feng, J. S. Lin, and L. C. Wang, "Green Resource Allocation Schemes for 
Relay-Enhanced MIMO-OFDM Networks", IEEE Transactions on Vehicular Technology, Vol.62, No.9, pp. 4539-4554, 2013.

[5] V. K. Y. Wu, Y. Li, M. P. Wylie-Green, T. Reid, and P. S. S. Wang, "Power allocation for OFDMbased cooperative relay systems", Journal of Communications and Networks, Vol.10, No.2, pp. 156-162, 2008.

[6] Y. Liu and M. Tao, "Optimal Channel and Relay Assignment in OFDM-Based Multi-Relay MultiPair Two-Way Communication Networks", IEEE Transactions on Communications, Vol.60, No.2, pp. 317-321, 2012

[7] Y. Jing and H. Jafarkhani, "Single and multiple relay selection schemes and their achievable diversity orders", IEEE Transactions on Wireless Communications, Vol.8, No.3, pp.1414-1423, 2009.

[8] K. Vardhe, D. Reynolds, and B. D. Woerner, "Joint power allocation and relay selection for multiuser cooperative communication", IEEE Transactions on Wireless Communications, Vol.9, No.4, pp.1255-1260, 2010.

[9] A. Bletsas, A. Khisti, D. P. Reed, and A. Lippman, "A simple Cooperative diversity method based on network path selection", IEEE Journal on Selected Areas in Communications, Vol.24, No.3, pp.659-672, 2006.

[10] B. Cao, X. Sun, Y. Li, C. Wang, and H. Mei, "Understanding the Impact of Employing Relay Node on Wireless Networks", IEEE Transactions on Vehicular Technology, Vol.66, No.5, pp.42874299, 2017.

[11] H. U. Sokun and H. Yanikomeroglu, "On the Spectral Efficiency of Selective Decode-andForward Relaying", IEEE Transactions on Vehicular Technology, Vol.66, No.5, pp.45004506, 2017.

[12] Z. Chen, P. Fan, and D. O. Wu, "Joint Power Allocation and Strategy Selection for HalfDuplex Relay System", IEEE Transactions on Vehicular Technology, Vol.66, No.3, pp.21442157, 2017.

[13] T. Q. Duong, T. M. Hoang, C. Kundu, M. Elkashlan, and A. Nallanathan, "Optimal Power Allocation for Multiuser Secure Communication in Cooperative Relaying Networks", IEEE Wireless Communications Letters, Vol.5, No.5, pp.516-519, 2016.

[14] W. Dang, M. Tao, H. Mu, and J. Huang, "Subcarrier-pair based resource allocation for cooperative multi-relay OFDM systems", IEEE Trans. Wireless Communication, Vol.9, No.5, pp.1640-1649, 2010.

[15] M. Hajiaghayi, M. Dong, and B. Liang, "Jointly Optimal Channel and Power Assignment for Dual-Hop Multi-Channel Multi-User Relaying",
IEEE Journal on Selected Areas in Communications, Vol.30, No.9, pp.1806-1814, 2012.

[16] X. Zhang, M. Hasna, and A. Ghrayeb, "Performance Analysis of Relay Assignment Schemes for Cooperative Networks with Multiple Source-Destination Pairs", IEEE Journal on Selected Areas on Wireless Communications, Vol.11, No.1, pp.166-177, 2012.

[17] Y. H. Tam, R. Benkoczi, H. S. Hassanein, and S. G. Akl, "Channel Assignment for Multihop Cellular Networks: Minimum Delay", IEEE Transactions on Mobile Computing, Vol.9, No.7, pp.1022-1034, 2010.

[18] R. Santhakumar and N. Amutha Prabha, "Robust Radio Resource Allocation in LTE Networks by Chanel and Relay Assignment", Journal of Engineering Science and Technology, Vol. 12, No. 7, pp. 1845 - 1854, 2017.

[19] V. Genc, S. Murphy, Y. Yu, and J. Murphy, "IEEE 802.16J relay-based wireless access networks: an overview", IEEE Wireless Communications, Vol.15, No.5, pp.56-63, 2008. 